\title{
Análisis y evaluación de la asociatividad de las comunidades en el mapa de influencia de la minería peruana mediante herramientas prospectivas - caso Ilabaya
}

Jorge Luis Inche Mitma* Alfonso Ramón Chung PinzÁs**

\section{INTRODUCCIÓN}

La asociatividad, entendida como instrumento de solución de los principales problemas que aquejan a un determinado colectivo, es una herramienta importante que permite la unión de los involucrados en el desarrollo de una comunidad, pueblo e incluso país. La relevancia de lograr un desarrollo cada vez más compartido, fijar objetivos comunes a corto, mediano y largo plazo, es una necesidad cada vez más patente, sobre todo en lugares en donde la pobreza es el común denominador de los actores.

Para el desarrollo de esta investigación, se abordó los problemas de las localidades ubicadas dentro del marco de influencia de las empresas mineras, en este caso se tomó una muestra y se analizaron los resultados; los cuales y debido a sus características, se podrá apreciar que son perfectamente extrapolables al resto de comunidades.

Sin embargo, el estudio no quedó en la presentación de los problemas, sino también en la búsqueda de soluciones mediante proyectos de desarrollo, los cuales se terminaron de formular y se presentaron a los organismos respectivos a fin de lograr su respectiva ejecución.

El problema que se estudió fue el siguiente: ¿Cómo lograr un desarrollo sostenible de las de las comunidades ubicadas dentro del marco de influencia de la minería?.

El presente artículo, muestra los resultados de una localidad y también presenta una metodología de consulta a expertos usada en la prospectiva, pero adaptada a un enfoque distinto; esta variación puede ser replicada en estudios similares en otras realidades.

\section{LA ASOCIATIVIDAD}

La asociatividad se puede definir como: "una estrategia para enfrentar los mercados globalizados y la creciente y fuerte competencia proveniente de otras empresas del país y del exterior" [9]; en esta definición se puede apreciar la importancia del trabajo conjunto de los entes involucrados, tanto para el

\footnotetext{
Doctor de Ciencias Administrativas. Profesor del Departamento de Diseño y Tecnología Industrial. UNMSM. E-mail: jlinche@hotmail.com

* Doctor en Ingeniería Industrial. Profesor del Departamento de Diseño y Tecnología Industrial. UNMSM. E-mail: ramon chung@yahoo.es
} 
mercado interno como externo y es justamente uno de los términos claves en cuanto a asociatividad, ya que también se define como "enfoque que reconoce la importancia estratégica del trabajo conjunto (articulado) entre las empresas" [4].

Si bien en la definición anterior no se hace hincapié en el ámbito de aplicación, se puede notar que coincide y refuerza lo propuesto por PROMPERÚ. Otro término clave es la estrategia, en ambos casos se puede ver que para ambas instituciones, la estrategia es también un punto clave en cuanto a asociatividad.

Porter [8] define a la estrategia como las acciones ofensivas o defensivas de una empresa para crear una posición defendible dentro de una industria; así pues aplicada esta definición a los conceptos de asociatividad analizados en los párrafos anteriores, se puede decir que la estrategia es el conjunto de acciones a realizar por los actores involucrados en alianza para el logro de un determinado objetivo. Sin embargo, es importante recordar que la estrategia se formula para lograr los objetivos a largo plazo [5].

Por lo tanto se puede afirmar que existen dos puntos clave en cuanto a la asociatividad, trabajo en conjunto y estrategia. Ahora, se hace necesario ubicar todos estos conceptos y términos en el ámbito de la investigación; para el enfoque propuesto, la asociatividad es la estrategia de trabajo conjunto de los actores involucrados en el desarrollo de un determinado colectivo. Esta estrategia de trabajo colectivo debe materializarse mediante la formulación y ejecución de proyectos de desarrollo sostenibles.

\section{PARTICIPACIÓN CIUDADANA}

Según la constitución Política del Perú, todo ciudadano tiene derecho a participar en forma individual o asociada en la vida política, económica y social de la nación; asimismo Ruelas [10] cita cuatro aspectos importantes en los cuales se soporta de participación ciudadana, sin embargo dos de ellos se sitúan dentro del marco de esta investigación:

a. Al incluir a la población habitualmente marginada de las decisiones, se promueve el empoderamiento ante los prestadores de servicios.

b. Exploración de nuevos enfoques de acción colectiva capaces de mostrar la racionalidad de la cooperación.
En ambos puntos, se resume claramente la importancia de la participación ciudadana, en el primero se puede apreciar que el ciudadano al tomar acción en las decisiones deja de ser un ser pasivo, asume su deber como actor del desarrollo y teniendo plena conciencia de sus deberes y derechos, puede no sólo fiscalizar sino también trabajar conjuntamente con el prestador de servicios en el desarrollo de su comunidad.

En el segundo caso, el trabajo con los involucrados, puede primeramente lograr un mejor conocimiento del problema que se va a resolver y al mismo tiempo novedosas formas de cómo resolverlo. En cuanto a la relación entre población y gobernantes, Pastor [7] establece la siguiente correspondencia entre objetivos de los ciudadanos y expectativas de las autoridades (véase Cuadro 1).

Cuadro 1. Correspondencia entre objetivos y espectativas

\begin{tabular}{|c|c|}
\hline $\begin{array}{l}\text { Objetivos } \\
\text { (ciudadanía) }\end{array}$ & $\begin{array}{l}\text { Expectativas } \\
\text { (Gobernantes) }\end{array}$ \\
\hline $\begin{array}{l}\text { Ser informados } \\
\text { Ser consultados } \\
\text { Tomar parte en las } \\
\text { decisiones }\end{array}$ & $\begin{array}{l}\text { Disponibilidad de } \\
\text { colaboradores } \\
\text { Legitimidad política } \\
\text { Mejora de las decisiones } \\
\text { Fortalecimiento del capital } \\
\text { social }\end{array}$ \\
\hline
\end{tabular}

Fuente: Pastor (2009), con modificaciones de los autores.

Cómo se puede apreciar en el cuadro anterior, es necesaria una relación de complementariedad entre la ciudadanía y los gobernantes, uno no es más que el otro, es decir los gobernantes no pueden abusar del poder transitorio que le ha dado el pueblo y este último no puede obstaculizar el trabajo del primero; asimismo esta relación debe estar orientada al desarrollo de la comunidad y al bien del país.

\section{EL ROL DE LOS ACTORES SOCIALES}

En 1968 un nuevo grupo de actores entrarían a escena: los jóvenes [2], ellos provistos de su energía y sus nuevas ideas harían ver a la sociedad conservadora de aquella época (la cual quizás no diste mucho de la actual), sus problemas y carencias, formándose diversos espacios de comunicación. Mediante este ejemplo se puede apreciar la importancia de la acción y el rol de los actores sociales, en este caso el ejemplo alude al movimiento de mayo del 68 , en la cual los jóvenes 
universitarios protagonizaron un papel importante en dicha gesta.

A lo largo de la historia los actores han jugado un rol preponderante, estos se pueden agrupar en clases sociales, partidos políticos entre otros [3]; en el Perú Haya de la Torre fue uno de los intelectuales que estudió a los actores agrupados en un frente único [6] contrario a la política de división de clases aplicada por la mayoría de intelectuales de izquierda marxista de aquella época.

Si bien el estado está compuesto de actores sociales y al mismo tiempo el estado es un escenario de lucha (ya sea entre ellos o contra el estado) a fin de lograr preservar sus intereses particulares [1]; es necesario lograr un punto medio de concertación mediante la asociatividad, proponiéndose objetivos comunes y trabajo en conjunto; es decir reorientar esta lucha.

\section{UBICACIÓN DE LAS LOCALIDADES ANALI- ZADAS}

Para el estudio se han considerado a expertos que en este caso son funcionarios de municipalidades, regionales y dirigentes gremiales; asimismo se ha trabajado en los siguientes lugares:

a. Distrito llabaya, provincia Jorge Basadre, Departamento Tacna

b. Ilo ciudad capital de la provincia de Ilo, departamento de Moquegua

c. Distrito Locumba, provincia Candarave, departamento de Tacna

Para el presente artículo, y por razones de espacio, se presentarán solamente los resultados del distrito de llabaya, pero el análisis fue similar en el resto de localidades.

\section{IDENTIFICACIÓN DE PROBLEMAS EN ILABAYA}

En este distrito, se formaron cuatro grupos de trabajo, los cuales identificaron diversos problemas, posteriormente se agruparon en una tabla de distribución para detectar las repeticiones que puedan existir; los resultados se muestran en el Cuadro 2.

Cuadro 2. Problemas agrupados y sus puntajes (llabaya)

\begin{tabular}{|c|c|c|c|c|c|c|c|c|}
\hline N. ${ }^{\circ}$ & Problema & $\begin{array}{l}0 \\
\text { 릉 } \\
\\
\end{array}$ & $\begin{array}{l}0 \\
\text { D } \\
\overline{0} \\
\mathrm{~N} \\
\mathrm{~N}\end{array}$ & 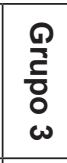 & 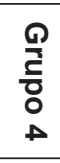 & $\stackrel{-1}{\stackrel{1}{1}}$ & $\frac{\circ}{\frac{0}{z}}$ & 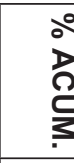 \\
\hline 1 & Recurso hídrico deficiente & 1 & 1 & 1 & 1 & 4 & $11 \%$ & $11 \%$ \\
\hline 2 & Disminución de frontera agrícola & 1 & & 1 & 1 & 3 & $8 \%$ & $18 \%$ \\
\hline 3 & $\begin{array}{l}\text { Mala cobertura de servicio de telecomunicaciones: telefonía fija, } \\
\text { móvil e internet en el distrito de llabaya }\end{array}$ & 1 & 1 & 1 & & 3 & $8 \%$ & $26 \%$ \\
\hline 4 & Conflictos territoriales entre pueblos vecinos & & 1 & 1 & 1 & 3 & $8 \%$ & $34 \%$ \\
\hline 5 & Contaminación & & 1 & 1 & 1 & 3 & $8 \%$ & $42 \%$ \\
\hline 6 & Congestionamiento vehicular en la vía de acceso llabaya capital & 1 & & 1 & & 2 & $5 \%$ & $47 \%$ \\
\hline 7 & Deficiente disposición final de aguas residuales y excretas. & & & 1 & 1 & 2 & $5 \%$ & $53 \%$ \\
\hline 8 & Mala utilización del recurso hídrico existente & & & 1 & & 1 & $3 \%$ & $55 \%$ \\
\hline 9 & $\begin{array}{l}\text { Daños en los terrenos agrícolas, zona urbana, infraestructura vial, } \\
\text { infraestructura de riego que ocasionan las avenidas en tiempo de } \\
\text { lluvias }\end{array}$ & & & 1 & & 1 & $3 \%$ & $58 \%$ \\
\hline 10 & Mala ubicación de centros poblados & & & 1 & & 1 & $3 \%$ & $61 \%$ \\
\hline 11 & $\begin{array}{l}\text { Desnutrición infantil en comunidades campesinas y centros } \\
\text { poblados en zona rural }\end{array}$ & & & 1 & & 1 & $3 \%$ & $63 \%$ \\
\hline 12 & $\begin{array}{l}\text { Problemas de plagas y enfermedades, en el cultivo de orégano, } \\
\text { ají, cebolla y alfalfa. }\end{array}$ & & & 1 & & 1 & $3 \%$ & $66 \%$ \\
\hline 13 & $\begin{array}{l}\text { Bajo rendimiento en la producción láctea y carne de la ganadería } \\
\text { vacuno en el distrito de llabaya. }\end{array}$ & & & 1 & & 1 & $3 \%$ & $68 \%$ \\
\hline 14 & No hay preparación de líderes profesionales del lugar & 1 & & & & 1 & $3 \%$ & $71 \%$ \\
\hline
\end{tabular}




\begin{tabular}{|l|l|l|l|l|l|l|l|}
\hline 15 & No hay corredor turístico con servicios & 1 & & 1 & $3 \%$ & $74 \%$ \\
\hline 16 & No hay atención medica permanente a la población & 1 & & & 1 & $3 \%$ & $76 \%$ \\
\hline 17 & No hay albergue para ancianos & 1 & & & 1 & $3 \%$ & $79 \%$ \\
\hline 18 & No hay guardería de niños en el distrito & 1 & & & 1 & $3 \%$ & $82 \%$ \\
\hline 19 & No hay optimo nivel educativo & 1 & & & 1 & $3 \%$ & $84 \%$ \\
\hline 20 & Reubicación de pozos sépticos & & 1 & & 1 & $3 \%$ & $87 \%$ \\
\hline 21 & Desastres naturales & 1 & & 1 & $3 \%$ & $89 \%$ \\
\hline 22 & Deficiente recaudación de tributos & 1 & & 1 & $3 \%$ & $92 \%$ \\
\hline 23 & $\begin{array}{l}\text { Deficiente desarrollo de la actividad agrícola debido al minifundio, } \\
\text { baja calidad agua y vulnerabilidad en las áreas agrícolas. }\end{array}$ & & & 1 & 1 & $3 \%$ & $95 \%$ \\
\hline 24 & $\begin{array}{l}\text { Deficiente fomento y atractivo a la inversión privada, para la } \\
\text { instalación de centros de acopio y transformación de los productos } \\
\text { primarios. }\end{array}$ & & & 1 & 1 & $3 \%$ & $97 \%$ \\
\hline 25 & $\begin{array}{l}\text { Deficientes niveles de desarrollo y uso de TIC y medios masivos } \\
\text { de información para la vinculación entre estado y sociedad. }\end{array}$ & & & 1 & 1 & $3 \%$ & $100 \%$ \\
\hline
\end{tabular}

Como se puede ver en el cuadro anterior, existen pocos problemas que tengan altas frecuencias, es decir la mayoría de opiniones no se concentran en uno o varios de estos problemas, sino más bien se diversifican; sin embargo, los siguientes problemas merecen principal atención debido a que han obtenido un mayor puntaje en la percepción de los expertos:

- Recurso hídrico deficiente.

- Disminución de frontera agrícola.

- Mala cobertura de servicio de telecomunicaciones: telefonía fija, móvil e internet en el distrito de llabaya.

- Conflictos territoriales entre pueblos vecinos.

- Contaminación.

- Congestionamiento vehicular en la vía de acceso llabaya capital.

- Deficiente disposición final de aguas residuales y excretas.

De estos el primero, debe de ser motivo de una acción inmediata. A continuación se presentan tres estadísticos que permiten conocer mejor las opiniones de la muestra (véase el Cuadro 3 ).

Cuadro 3. Principales estadísticos

\begin{tabular}{|l|l|}
\hline Promedio & 1.5 \\
\hline Desviación estándar & 0.9 \\
\hline Mediana & 1 \\
\hline
\end{tabular}

El promedio está cercano a 1, es decir una repetición por cada problema, asimismo la desviación estándar indica que no existe una desviación alta entre los datos con respecto al promedio y finalmente la mediana también muestra que el número 1 es el que divide a todos los datos en la mitad.

Por otro lado, el Cuadro 4 muestra las frecuencias agrupadas por puntaje, en donde se puede apreciar que el $72 \%$ de los datos tiene como puntaje 1, es decir una observación; lo cual es un indicativo de que no hay mucha repetición en cuanto a los problemas, es decir no existe una percepción común de los mismo problemas.

Cuadro 4. Agrupación por puntajes

\begin{tabular}{|c|c|c|c|}
\hline Puntaje & Frec. & $\begin{array}{c}\text { Porcentaje } \\
\text { individual }\end{array}$ & $\begin{array}{c}\text { Porcentaje } \\
\text { acumulado }\end{array}$ \\
\hline 1 & 18 & $72 \%$ & $72 \%$ \\
\hline 3 & 4 & $16 \%$ & $88 \%$ \\
\hline 2 & 2 & $8 \%$ & $96 \%$ \\
\hline 4 & 1 & $4 \%$ & $100 \%$ \\
\hline TOTAL & 25 & $100 \%$ & \\
\hline
\end{tabular}

A continuación se presentan los proyectos de asociatividad seleccionados por los expertos, considerando la factibilidad de la solución de algunos problemas de los cuadros anteriormente presentados. Es importante mencionar que estos proyectos han sido desarrollados bajo la metodología SNIP, sino 
que por razones de espacio, sólo se mencionan los títulos, ya que su desarrollo sería motivo de otro proyecto de investigación.

\section{PROYECTOS DE SOLUCIÓN IDENTIFICADOS}

Los proyectos de identificados por los expertos están enfocados a los siguientes problemas:

\section{- Recurso hídrico deficiente}

- Contaminación

- Congestionamiento vehicular en la vía de acceso Ilabaya capital
- Deficiente disposición final de aguas residuales y excretas.

- No hay atención médica permanente a la población

- Desastres naturales

- Deficiente desarrollo de la actividad agrícola debido al minifundio, baja calidad agua y vulnerabilidad en las áreas agrícolas.

El Cuadro 5 muestra los proyectos junto con los problemas que los originan.

Cuadro 5. Problemas y proyectos (Ilabaya)

\begin{tabular}{|c|c|c|}
\hline N. ${ }^{\circ}$ & Problema & Proyecto \\
\hline 1 & Recurso hídrico deficiente & $\begin{array}{l}\text { Mejoramiento y ampliación del sistema de agua potable e } \\
\text { instalación de sistema de alcantarillado en la localidad de } \\
\text { Higuerani, Distrito de llabaya - Jorge Basadre - Tacna }\end{array}$ \\
\hline \multirow{2}{*}{2} & \multirow{2}{*}{$\begin{array}{l}\text { Congestionamiento vehicular en la vía de acceso } \\
\text { llabaya capital }\end{array}$} & $\begin{array}{l}\text { Mejoramiento de vías urbanas en el centro poblado de } \\
\text { Mirave, distrito de llabaya, provincia de Jorge Basadre- } \\
\text { region Tacna }\end{array}$ \\
\hline & & $\begin{array}{l}\text { Mejoramiento de las calles en el barrio de San Bartolomé } \\
\text { del c.p.m. Borogueña, distrito de llabaya, provincia Jorge } \\
\text { Basadre, departamento de Tacna }\end{array}$ \\
\hline 3 & $\begin{array}{l}\text { Deficiente disposición final de aguas residuales } \\
\text { y excretas. }\end{array}$ & $\begin{array}{l}\text { Mejoramiento y ampliación del sistema de agua potable e } \\
\text { instalación de sistema de alcantarillado en la Localidad de } \\
\text { Higuerani, Distrito de llabaya - Jorge Basadre - Tacna }\end{array}$ \\
\hline 4 & $\begin{array}{l}\text { No hay atención medica permanente a la } \\
\text { población }\end{array}$ & $\begin{array}{l}\text { Mejorar la capacidad resolutiva de los servicios de salud en } \\
\text { el Centro de Salud Pocollay }\end{array}$ \\
\hline \multirow{2}{*}{5} & \multirow{2}{*}{ Desastres naturales } & $\begin{array}{l}\text { Construcción de defensas y encauzamiento en las } \\
\text { quebradas Ticapampa y Gallinazos, en la localidad de } \\
\text { Ticapampa, distrito de llabaya, provincia Jorge Basadre- } \\
\text { Tacna }\end{array}$ \\
\hline & & $\begin{array}{l}\text { Construcción de defensas contra avenida en las quebradas } \\
\text { de incidencia al centro poblado de mirave, distrito de } \\
\text { ilabaya, provincia Jorge Basadre-Tacna", distrito de llabaya, } \\
\text { provincia Jorge Basadre - Tacna }\end{array}$ \\
\hline 6 & $\begin{array}{l}\text { Deficiente desarrollo de la actividad agrícola } \\
\text { debido al minifundio, baja calidad agua y } \\
\text { vulnerabilidad en las áreas agrícolas. }\end{array}$ & $\begin{array}{l}\text { Asistencia técnica y capacitación en la producción del } \\
\text { orégano en los C.P. de Borogueña y Cambaya del distrito } \\
\text { de llabaya }\end{array}$ \\
\hline
\end{tabular}




\section{CONCLUSIONES}

a. Es factible la aplicación de metodologías prospectivistas, para la detección de problemas sociales.

b. Para lograr una efectiva participación de la ciudadanía en la búsqueda de soluciones es necesaria una capacitación adecuada reforzando los deberes y derechos de cada poblador.

c. Los proyectos de asociatividad son factibles y necesarios para la solución de los problemas en las zonas de estudio.

d. La formulación de cualquier proyecto de mejora debe pasar necesariamente por la consulta a involucrados y a expertos.

\section{REFERENCIAS BIBLIOGRÁFICAS}

[1] Baca L. (2000). Léxico de la política. CONACYT. México

[2] Bolos S. (1999). La Constitución de Actores Sociales y la Política. Universidad Iberoamericana. México.
[3] Cohen E. y Franco R. (2000). Evaluación de Proyectos Sociales.Siglo XXI. México

[4] FIAC (2006). Asociatividad. En: http://www. mincetur.gob.pe

[5] Hax A. y Majluf N. (2004). Estrategias para el liderazgo competitivo. Ediciones Granica S.A. Argentina.

[6] Haya V. (1926). El Frente Único. Extraído de: http://upgp.tripod.com/id20.html

[7] Pastor E. (2009). Participación ciudadana y gestión de las políticas sociales municipales. EDITUM. España.

[8] Porter M. (2008). Competitive Strategy: Techniques for Analyzing Industries and Competitors. Simon and Schuster. USA

[9] PROMPERÚ (2006). Asociatividad. En: http:// www.promperu.gob.pe/

[10]Ruelas E. (2008). Participación ciudadana en la mejora de la calidad de los servicios de salud. Ed. Médica Panamericana. México. 